DEPÓSITO LEGAL ZU2020000153

Esta publicación científica en formato digital

es continuidad de la revista impresa

ISSN 0041-8811

E-ISSN 2665-0428

Revista

de la

Universidad

del Tunlia

Fundada en 1947

por el Dr. Jesús Emrique Lossada

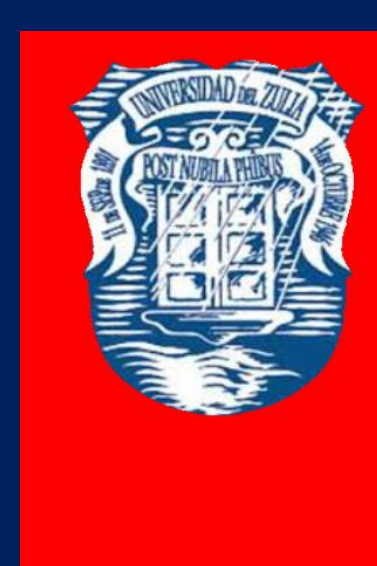

Ciencias

Sociales

y Arte

Aกัต 11 No 31

Septiembre - Diciembre 2021

Tercera ípoca

Maracailbo-Venezuela 
REVISTA DE LA UNIVERSIDAD DEL ZULIA. 3época. Año $11 \mathrm{~N}^{\circ}$ 31, 2020 Arnaldo Vergara-Romero et al. /// Factores de desarrollo local desde el enfoque ...86-96

DOI: http://dx.doi.org/10.46925//rdluz.31.07

\title{
Factores de desarrollo local desde el enfoque de la gestión organizativa
}

\author{
Arnaldo Vergara-Romero * \\ Rafael Sorhegui-Ortega ** \\ Lisette Garnica-Jarrin ***
}

\section{RESUMEN}

La evolución del desarrollo local, como eje estratégico para el desarrollo de un país, es el primer componente que está inmerso en el discurso político y en las normativas de carácter regulador en el territorio ecuatoriano, el cual tiene convenios firmados con organismos multilaterales internacionales. El siguiente articulo analiza los factores sociales de la formación de la comunidad local como un sistema social y la gestión de las autoridades de dicha comunidad. Se utilizó como metodología el diseño fenomenológico hermenéutico y como instrumento se utilizó la observación documental de artículos científicos de revistas indexadas en Web of Science y Scopus. Se concluye que se necesita una mejora del sistema de gobiernos locales, del desarrollo de los recursos mencionados y de las oportunidades para apoyar la actividad social.

PALABRAS CLAVE: autoorganización, desarrollo endógeno, desarrollo local, desarrollo sostenible, desarrollo territorial.

* Docente. Universidad Espíritu Santo, Samborondón, Ecuador. avergarar@ecotec.edu.ec, https://orcid.org/0000-0001-8503-3685

** Docente. Universidad Ecotec, Samborondón, Ecuador. rsorhegui@ecotec.edu.ec https://orcid.org/0000-0001-7882-5246

*** Investigadora, Daule, Ecuador. E-mail: lisg13@gmail.com

Recibido: 16/06/2020

Aceptado: 31/07/2020 
REVISTA DE LA UNIVERSIDAD DEL ZULIA. 3época. Año 11 Nº 31, 2020 Arnaldo Vergara-Romero et al. /// Factores de desarrollo local desde el enfoque ...86-96

DOI: http://dx.doi.org/10.46925//rdluz.31.07

\section{Local development factors from the organizational management perspective}

ABSTRACT

The evolution of local development, as a strategic axis for the development of a country, is the first component involved in the political speech and in regulatory protocols in the Ecuadorian territory, which has signed agreements with international multilateral organizations. The following article analyzes the social factors of local community formation as a social system and the management of the authorities of such community. Hermeneutic phenomenological design was used as a methodology, and documentary observation of scientific articles from journals indexed in Web of Science and Scopus was used as an instrument. As conclusion, it is indispensable the improvement of the local government system, the development of the studied resources and the opportunities to support social activity.

KEYWORDS: self-organization, endogenous development, local development, sustainable development, territorial development.

\section{Introducción}

La importancia de involucrar a las comunidades territoriales en el gobierno central es innegable. El Presidente de la República del Ecuador, Lenin Moreno, anunció que se va "robustecer la integración regional y apoyar las iniciativas y espacios que la potencien" (Moreno, 2017, p. 18), destacando la importancia de los territorios en la planificación gubernamental.

Esta sugestión encuentra su contraparte legal en el Plan Nacional de Desarrollo 2017 2021, cuya directrices y lineamientos territoriales en su sección-d, indica que se debe "planificar el desarrollo económico sobre la base de la compatibilidad de usos y la vocación territorial, sus diversidades culturales y sus dinámicas socio-ambientales", creando un "impulso a la productividad y la competitividad sistemática a partir del potenciamiento de los roles y funcionalidades del territorio" (SENPLADES, 2017, p. 124).

De igual manera, la agenda 2030 sobre el Desarrollo Sostenible, incluye el Objetivo de Desarrollo número 1l, que menciona: “Apoyar los vínculos económicos, sociales y ambientales positivos entre las zonas urbanas, periurbanas y rurales fortaleciendo la planificación del desarrollo nacional y regional" (UN, 2020). 
REVISTA DE LA UNIVERSIDAD DEL ZULIA. 3épeca. Año 11 N³1, 2020 Arnaldo Vergara-Romero et al. /// Factores de desarrollo local desde el enfoque ...86-96

DOI: http://dx.doi.org/10.46925//rdluz.31.07

El siguiente articulo analiza los factores sociales de la formación de la comunidad local como un sistema social y la gestión de las autoridades de dicha comunidad. Destacando la importancia de involucrar a todos los actores que diseñan tanto alternativas como en la sociedad civil y políticas públicas por parte del gobierno local.

\section{Metodología}

Para poder cumplir el objetivo del artículo, este se fundamentó en el diseño fenomenológico hermenéutico. Se estructura desde el estudio y la reflexión del fenómeno, descubriendo categorías o factores para continuamente describirlo (Dubinina, 2019). El instrumento que se utilizó fue la observación documental de artículos científicos, en revistas que utilizan el método "por pares doble ciego" (Schonhaut Berman et al, 2017) e indexadas en Web of Science y Scopus, para garantizar el rigor científico.

\section{Entendiendo el desarrollo local}

Para entender al desarrollo local se debe analizar su propósito, el cual es desarrollar la capacidad de un territorio definido para mejorar su futuro económico y la calidad de vida de los habitantes (Gori y Sodini, 2020).

Por lo tanto, el desarrollo local hace una contribución importante al desempeño nacional y se vuelve más critico con la globalización, la movilidad de personas y los avances tecnológicos, trayendo consigo reducir las disparidades entre los lugares que tienen poco desarrollo y las que tienen mejor desarrollo (Kisman y Tisar, 2014).

Para León Segura y Sorhegui Ortega (2007), “el desarrollo local exige una planificación y una dirección consciente del proceso y desarrollo económico local, esto supone elaborar una política de desarrollo local" (p. 160). Asimismo, explican que los "factores condicionantes para el potencial de desarrollo local endógeno son: recursos materiales, recursos humanos, recursos técnicos, recursos socioculturales y por último la capacidad de organización local" (p.160).

Según Sekula (2002), señala que el desarrollo local es un proceso continuo, que este se refiere a un área y, por lo tanto, es un desarrollo territorial, no un desarrollo territorial, provocando un sentimiento de pertenencia a una comunidad. También destaca que la iniciativa, la creatividad y la inventiva de las entidades locales dependen en gran medida de 
REVISTA DE LA UNIVERSIDAD DEL ZULIA. 3a época. Año 11 N³ 31, 2020 Arnaldo Vergara-Romero et al. /// Factores de desarrollo local desde el enfoque ...86-96

DOI: http://dx.doi.org/10.46925//rdluz.31.07

la conciencia de que pueden influir en la dirección del desarrollo y las autoridades locales desempeñan un papel incuestionable en el desarrollo local (Sili, 2019).

3. La gestión organizativa

Los gobiernos locales son considerados como la unidad más pequeña de representación político-territorial, estos gobiernos habitualmente se los denomina “Administración local”, ya que tiene un gran papel representativo y responsabilidades en el ámbito económico, social y administrativo (Porras Ramírez, 2005).

Partiendo de esta premisa, se puede entender la gran expansión del término descentralización de las responsabilidades gubernamentales y su avance práctico en el mundo como parte de la gestión organizativa. El principio rector de la descentralización es la delegación, la cual implica jurisdicciones subnacionales independientes a las que se le asignas responsabilidades de prestación de servicios públicos, pero sujetos a la supervisión del gobierno central con respecto al nivel y calidad del servicio que se prestará, debidamente financiado (Zsamboki y Bell, 1997).

La gestión organizativa es siempre vista por su enfoque de autogobierno, que conduce a una mejor coordinación de la organización de actividades y una mejor utilización de equipos técnicos y personal. De igual manera se encuentra la autosuficiencia y la eficiencia en la prestación de servicios públicos, que a menudo está relacionado con el valor al bienestar social (Willmott, 2019).

\section{Desarrollo local y su autogobierno}

Es indudable que cualquier ciudad o parroquia puede desarrollarse en un entorno de éxito con los residentes de dicha comunidad, basados en su opinión, sus iniciativas, con instituciones fuertes, democracia directa, mecanismos eficaces de comunicación y un buen gobierno local (Kim, 2020).

El nivel territorial es de particular importancia a la luz de los problemas de optimización de los procesos de gestión a nivel de una comunidad parroquial, cantonal, provincial o regional. El desarrollo de las comunidades locales está influenciado por una serie de factores sociales que tienen diferentes influencias en el estado sociocultural, económico y 
REVISTA DE LA UNIVERSIDAD DEL ZULIA. 3a época. Año 11 N³ 31, 2020 Arnaldo Vergara-Romero et al. /// Factores de desarrollo local desde el enfoque ...86-96

DOI: http://dx.doi.org/10.46925//rdluz.31.07

político vigente en la sociedad, directamente relacionados con la eficacia de la gestión municipal (Boone y Péli, 2016).

El desarrollo teórico del análisis de la naturaleza y las características de las comunidades locales, permiten justificar el estudio de las actividades grupales, el sistema de relaciones públicas, y también considerar a la comunidad local a través del prisma de los hitos de la gestión municipal (Nikula y Kopoteva, 2020).

El comportamiento de la población para resolver problemas de la comunidad local está influenciado por factores objetivos y subjetivos de la actividad transcendental de los ciudadanos. A pesar de la gran variedad de publicaciones, es destacable señalar que en ellas se considera a la comunidad local fundamentalmente desde le punto de vista de la sociología, política, economía y el derecho (Tomashuk, 2017; Misakov et al., 2020).

Al mismo tiempo, el enfoque sistémico-sociológico para el análisis de la comunidad local sigue siendo relevante, y la comunidad local, como sistema social, no ha sido suficientemente estudiada. Para ello, se destacan las siguientes propiedades sistémicas de la comunidad local:

- $\quad$ Residencia compacta de la población en un territorio determinado, designado por límites geográficos, económicos, ambientales y de otros tipos (Rubiales Pérez, 2020).

- La disponibilidad de recursos de la comunidad territorial para satisfacer las necesidades de la población (Griffiths et. al., 2020).

- $\quad$ Reproducción del grupo territorial de la población, el entorno de vida debido a los recursos internos y externos del sistema como una capacidad sostenible (Marošević y Bošnjak, 2018).

- Identificación y autoidentificación de residentes, conciencia de su pertenencia a la comunidad local (Golikov et al, 2018).

- La presencia de necesidades comunes o cercanas en la organización de la vida (Ikeanyibe, 2018).

- El desarrollo de elementos de autogobierno o autogestión (Frolova et al., 2020).

Se puede argumentar que la comunidad local es un sistema social incorporado que está unido por el territorio de residencia permanente o predominante, capaz de autoconservación y desarrollo, como sujeto de gestión y mejora de la calidad de vida humana, como el eslabón principal del sistema (Dvoryadkina y Belousova, 2020). 
REVISTA DE LA UNIVERSIDAD DEL ZULIA. 3a época. Año 11 N³ 31, 2020 Arnaldo Vergara-Romero et al. /// Factores de desarrollo local desde el enfoque ...86-96

DOI: http://dx.doi.org/10.46925//rdluz.31.07

Por lo tanto, la comunidad local se considera, en primer lugar, como una sociedad civil, la base para la autoorganización territorial y el autogobierno de los ciudadanos, y en segundo lugar, como un tema de gestión social del espacio territorial (Tolkovanov, 2017).

La actividad de la comunidad local está determinada por la interacción de los ciudadanos y los organismos de autogobierno público territorial como entidades de gestión (actores sociales) que resuelven directamente los problemas de mejora en materia de la calidad de vida (Barkhatov et al., 2019). El grado de participación de la población en la resolución de problemas de gobernanza local se considera un indicador de la madurez de la comunidad local (Car-Pušić, Marović y Bulatović, 2019).

Sin embargo, en la práctica se muestra que la población es indiferente a las actividades de las autoridades locales. Las principales razones de esta contradicción en la interacción entre las autoridades y las comunidades locales son: el equilibrio de intereses y la falta de confianza entre los actores de la gobernanza social (Mazur y Tomashuk, 2019).

Es importante tener en cuenta las condiciones socioculturales de la realidad ecuatoriana y la calidad de vida, esta última tiene un impacto significativo en el crecimiento o declive de la comunidad local de municipios (Scaramuzzi, Belletti y Biagioni, 2020). La formación de las comunidades locales esta ciertamente influenciada por la calidad del desempeño de las funciones de poder, especialmente el paternalismo de las autoridades en relación con la población, el distanciamiento de la misma y el cierre de las actividades (Marošević y Bošnjak, 2018).

Teniendo en cuenta la clasificación de los factores que afectan directamente a las peculiaridades de la actividad poblacional en las comunidades locales, propuestas en este articulo, así como los enfoques anteriores de los indicadores de actividad, se destacarán factores objetivos y subjetivos, incluyendo cuestiones económicas, políticas, culturales, demográficas y organizativas (Korneć, 2020).

Los factores objetivos incluyen cuestiones a nivel nacional, legales y políticas que tienen un impacto común en la nación y la actividad de la comunidad local (Colomb y Tomaney, 2020). Esto también incluye factores locales: económico, geográfico, histórico, demográfico, cultural, étnico y gerencial. Su influencia es constante, la dinámica del estado es estable, los cambios reales son posibles sólo con un impacto sistémico en la gestión más a menudo regional que a nivel local (Frolova et al., 2020). 
REVISTA DE LA UNIVERSIDAD DEL ZULIA. 3época. Año 11 Nº 31, 2020 Arnaldo Vergara-Romero et al. /// Factores de desarrollo local desde el enfoque ...86-96

DOI: http://dx.doi.org/10.46925//rdluz.31.07

Al mismo tiempo, existen algunos investigadores que observan que los factores objetivos causan contradicciones entre las capacidades de recursos de los territorios, el potencial humano y la baja eficiencia del sistema de gobierno local (Amendola et al., 2020). La consecuencia negativa de la acción de factores objetivos es la reducción del número de comunidades sociales y territoriales, su unificación y eliminación, que provoca la migración y el declive de la población (Gordon y Kourtit, 2020).

Conjuntamente, el crecimiento económico regional provocaría procesos inversos, es muy imperativo que el emprendimiento también es un factor para aumentar la actividad de la población y desarrollar la comunidad local (Turley y McNena, 2019).

Los factores subjetivos incluyen la situación política y la credibilidad de líderes locales específicos, la eficacia de sus actividades, el grado de confianza pública, el espacio de información de interacción con los ciudadanos, los canales de dicha interacción, incluida la retroalimentación. Algunos factores son interdependientes, por lo que la apertura de información de la comunidad, es una de las principales condiciones para aumentar la confianza del público (Tran y Dollery, 2020).

Es muy significativo resaltar que, la gran parte de los ciudadanos tiene poca conciencia de las actividades de los gobiernos locales y de la falta de información en el dominio público. El diálogo con las autoridades es igualmente importante para resolver el problema de la confianza pública (Pike et al., 2013). La pasividad de los gobiernos locales, la disuasión de las iniciativas ciudadanas, la espera de soluciones de la autoridades nacionales y provinciales, el cultivo de la dependencia entre la población también dificulta la revitalización de la comunidad local (Galiullina et al, 2018). Por lo tanto, uno de los factores subjetivos más importantes es el problema del personal profesional calificado en el gobierno local.

Dentro del razonamiento se observa que para formar una comunidad local eficaz y funcional se requiere lo siguiente:

- Los residentes deben tener intereses comunes en los problemas locales más apremiantes.

- Los intereses no sólo deben entenderse, sino también reforzarse con la voluntad de aplicarlos realmente.

- Los formularios y métodos de implementación deben ser reales. 
REVISTA DE LA UNIVERSIDAD DEL ZULIA. 3épeca. Año 11 N³1, 2020 Arnaldo Vergara-Romero et al. /// Factores de desarrollo local desde el enfoque ...86-96

DOI: http://dx.doi.org/10.46925//rdluz.31.07

- El colectivo se encargará de abordar los problemas a los que se enfrenta la comunidad por sí misma y responsable.

\section{Conclusión}

Los detalles de la formación de la comunidad local, los factores de influencia determinan la existencia y el desarrollo de la comunidad local. La comunidad local puede y debe convertirse en un actor real en la sociedad civil ecuatoriana. La tarea de las organizaciones públicas y las autoridades de gobiernos locales es activar factores objetivos, subjetivos y las formas de autoorganización para resolver los problemas locales.

También se necesita una mejora del sistema de gobiernos locales, el desarrollo de los recursos mencionados y las oportunidades para apoyar la actividad social. Una mayor participación de la población en la preparación de soluciones para las cuestiones de la comunidad local es posible mediante el uso de tecnologías modernas de comunicación de la información, la revitalización del interés de los ciudadanos en las audiencias públicas y la formación de mecanismos para la implementación de iniciativas locales.

\section{Referencias}

Amendola, A., Barra, C. y Zotti, R. (2020). Does graduate Human Capital Production Increase Local Economic Development? An Instrumental Variable Approach. Journal Regional Science. Doi: 10.1111/jors.12490.

Barkhatov, V. I., Kapkaev, Y. S., Bents, D. S., Kadyrov, P. R. e Isaeva, A. (2019). Economic Growth of Russian Regions: Condition, Factors, Opportunities. The International Scientific and Practical Conference "Contemporary Issues of Economic Development of Russia: Challenges and Opportunities”, 59, 430-444. Doi: 10.15405/epsbs.2019.04.47

Boone, C. y Péli, G. (2016). Globalization and variants of local adaptation: Theory and justification with symbolic logic. International Sociology, 1-24. Doi: 10.1177/0268580916662384

Car-Pušić, D., Marović, I. y Bulatović, G. (2019). Agile Organizational Model for managing Local Government projects. Electronic Journal of the Faculty of Civil Engineering OSIJEKE-GFOS, 18, 12-21. Doi: 10.13167/2019.18.2

Colomb, C. y Tomaney, J. (2020). Spatial planning, nationalism and territorial politics in Europe. Regional Studies. Doi: 10.1080/00343404.2020.1744552

Dubinina, V. O. (2019). Methodology of Philosophical Hermeneutics in the Social Sciences. Science Review, 2(19), 25-31. Doi: 10.31435/rsglobal_sr/28022019/6366 
REVISTA DE LA UNIVERSIDAD DEL ZULIA. 3a época. Año 11 N³1, 2020 Arnaldo Vergara-Romero et al. /// Factores de desarrollo local desde el enfoque ...86-96

DOI: http://dx.doi.org/10.46925//rdluz.31.07

Dvoryadkina, E. B. y Belousova, E. A. (2020). Trends of the Development of Municipal Regions in the National Economic Space. Economic and Social Changes: Facts, Trends, Forecast, 13(1), 87-105. Doi: 10.15838/esc.2020.1.67.5

Frolova, E., Rogach, O., Ryabova, T. y Morozov, V. (2020). Political and economic autonomy of local self-government as a factor of social infrastructure development in Russia. International Journal of Advanced and Applied Sciences, 7(3), 64-74. Doi: 10.21833/ijaas.2020.03.008

Galiullina, G., Sharamko, M. y Mironova, T. (2018). The concept of creating territories of advanced socio-economic development. Amazonia Investiga, 7(17), 371-377. https://www.amazoniainvestiga.info/index.php/amazonia/article/view/743

Golikov, S., Korneyko, O. y Krasova, E. (2018). The strategy of territory socio-economic image formation. Amazonia Investiga, $262-270$. https://www.amazoniainvestiga.info/index.php/amazonia/article/view/456

Gordon, P. y Kourtit, K. (2020). Agglomeration and clusters near and far for regional development: A critical assessment. Regional Science Policy and practice, 1-10. Doi: $10.1111 / \mathrm{rsp} 3.12264$

Gori, L. y Sodini, M. (2020). Endogenous labour supply, endogenous lifetime and economic development. Structural Change and Economic Dynamics, 52, 238-259. Doi: 10.1016/j.strueco.2019.11.006

Griffiths, V. F., Bull, J. W., Baker, J., Infield, M., Roe, D., Nalwanga, D., Byaruhanga, A. y Milner-Gulland, E. J. (2020). Incorporating local nature-based cultural values into biodiversity No Net Loss Strategies. World Development, 128(104858), 1-15. Doi: 10.1016/j.worlddev.2019.104858

Ikeanyibe, O. M. (2018). Uniformity in Local Government system and the Government System and the Governance Model in Nigeria. Journal of Asian and African Studies, 53(1), 147-161. Doi: 10.1177/0021909616666105

Kim, S. (2020). Inter-municipal relations in city-region governance. Cities, 104(102771), 1-10. Doi: 10.1016/j.cities.2020.102771

Kisman, Z. A. y Tasar, I. (2014). The key elements of local development. Procedia Economics and Finance, 15, 1689 - 1696. Doi: 10.1016/S2212-5671(14)00642-X

Korneć, R. (2020). The Role of Stakeholders in Shaping Smart Solutions in Polish Cities. Entrepreneurship and Sustainability Issues, 7(3), 1981-1995. Doi: 10.9770/jesi.2020.7.3(36)

León Segura, C. M. y Sorhegui Ortega, R. (2007). Repensar el desarrollo local en Cuba. Economía y Desarrollo, 142(2), 150-171. https://www.redalyc.org/pdf/4255/425541311008.pdf

Marošević K. y Bošnjak, D. (2018). Regional Development and Glocalisation: Theoretical Framework. Economski Vjesnik, 31(1), 201-213. 
REVISTA DE LA UNIVERSIDAD DEL ZULIA. 3épeca. Año 11 N³1, 2020 Arnaldo Vergara-Romero et al. /// Factores de desarrollo local desde el enfoque ...86-96

DOI: http://dx.doi.org/10.46925//rdluz.31.07

Mazur, K. y Tomashuk, I. (2019). Governance and Regulation as an indispensable condition for developing the potential of rural areas. Baltic Journal of Economic Studies, 5(5), 67-78. Doi: 10.30525/2256-0742/2019-5-5-67-78

Misakov, Anzor V; Sabanchiev, Anzor Kh.; Sanginova, Lola D.; Danilevskaya, Elena N.; Eskiev, Musa A. (2020). Assessment of the main reasons for the stagnation of territorial development in Russia, Revista de la Universidad del Zulia, 11 (29), 254-264. DOI: http://dx.doi.org/10.46925//rdluz.29.16

Moreno, L. (2017). Discurso de posesión del Presidente Lenín Moreno Garcés ante la Asamblea nacional en presencia de jefes de estado, delegaciones e invitados nacionales e internacionales. Recuperado de https:/www.presidencia.gob.ec/wpcontent/uploads/downloads/2017/06/2017. 05 .24-DISCURSO-POSESI\%C3\%93N-ANTELA-ASAMBLEA-NACIONAL.pdf

Nikula, J. y Kopoteva, I. (2020). Local development initiatives in the Lake Ladoga Region: Reasons for success and approaches to funding. RUDN Journal of Sociology, 20(1), 102-114. Doi: $10.22363 / 2313-2272-2020-20-1-102-114$

Porras Ramírez, J. M. (2005). El autogobierno local en el Estado autonomico. Premisas para una reforma necesaria. Revista Española de Derecho Constitucional, 75, 211-237. https://www.jstor.org/stable/24885247

Pike, A., Rodríguez-Pose, A. y Tomaney, J. (2013). Local and regional development in the global North and South. Progress in Development Studies, 14(1), 21-30. Doi: $10.1177 / 1464993413504346$

Rubiales Perez, M. (2020). Segregación en las metrópolis españolas 2001-2011: un analisis con detalle territorial. Documents d'Anàlisi Geogràfica, 66(1), 83-105. Doi: 10.5565/rev/dag.581

Scaramuzzi, S., Belletti, G. y Biagioni, P. (2020). Integrated Supply Chain Projects and multifunctional local development: the creation of a Perfume Valley in Tuscany. Agricultural and Food Economics, 8(5), 1-16. Doi: 10.1186/s40100-019-0150-8

Schonhaut Berman, L., Millán Klusse, T. y Podestá López, L. (2017). Revisión por pares: evidencias y desafios. Revista Chilena de Pediatria, 88(5), 577-581. Doi: http://dx.doi.org/10.4067/S0370-41062017000500001

Sekula, A. (2002). Local development - the definition aspect in the 21st century. Company at the Turn of the $21^{\text {st }}$ Century, Politechnika Rzeszowska. 59-64.

SENPLADES. (2017). Plan Nacional de Desarrollo 2017-2021. Secretaria Nacional de Planificación y Desarrollo. Recuperado de https://www.planificacion.gob.ec/wpcontent/uploads/downloads/2017/10/PNBV -26-OCT-FINAL_OK.compressedl.pdf

Sili, M. (2020). Acción territorial y construcción del desarrollo. La experiencia de zonas rurales de la Pampa argentina. Revista de Geografía Norte Grande, 74, 159-186. Doi: 10.4067/S071834022019000300159 
REVISTA DE LA UNIVERSIDAD DEL ZULIA. 3épeca. Año 11 N³1, 2020 Arnaldo Vergara-Romero et al. /// Factores de desarrollo local desde el enfoque ...86-96

DOI: http://dx.doi.org/10.46925//rdluz.31.07

Tolkovanov, V. V. (2017). Territorial Communities' Cooperation (Intermunicipal Cooperation - IMC) as an innovative tool for ensuring local economic development and municipal consolidation. Scientific Bulletin of Polissia, 1, 76-80.

Tomashuk, I. (2017). Problems and Prospects of Management of Rural Development. Baltic Journal of Economic Studies, 3(5), 214-220. Doi: 10.30525/2256-0742/2017-3-5-214-220

Tran, C-D. T. T. y Dollery, B. (2020). An empirical analysis of municipal efficiency and local resident satisfaction by council type in Victorian local government. Australian Journal of Public Administration, Doi: 10.1111/1467-8500.12421

Turley, G. y McNena, S. (2019). Local government funding in Ireland: Contemporary issues and future challenges. Administration, 67(4), 1-26. Doi: 10.2478/admin-2019-0024

UN. (2020). United Nations. Objetivos de Desarrollo Sostenible. Recuperado el 10 de enero de 2020 de https://www.un.org/sustainabledevelopment/es/

Willmott, K. (2019). From self-government to government of the self: Fiscal subjectivity, Indigenous governance and the politics of transparency. Critical Social Policy, 40(3), 471-491. Doi: https://doi.org/10.1177/0261018319857169

Zsamboki, K. y Bell, M. (1997). Local Self-Government in Central and Eastern Europe: Decentralization or Deconcentration? Environment and Planning C: Politics and space, 15(2), 177-186. Doi: https://doi.org/10.1068/c150177 